\title{
Model Analysis of Car Five Degree of Freedom Vibration System Based on Energy Decoupling Method
}

\author{
XU Bo ${ }^{1, a}$, BAO Jiading ${ }^{2,3, b, *}$, WU Jianwei ${ }^{4, c}$, WANG Hanchao ${ }^{5, d}$ \\ ${ }^{1,2,4,5}$ Guilin University of Electronic Technology, Guilin 541004, China \\ ${ }^{3}$ Guangxi Key Laboratory of Manufacturing Systems and Advanced Manufacturing Technology, \\ Guilin 541004, China \\ a154751099@qq.com, ${ }^{\mathrm{C}}$ wujianwei081x@163.com, ${ }^{\mathrm{d} w h c 120005 @ 126 . c o m ~}$ \\ ${ }^{b, \star}$ Communication author: jdbaoguet@163.com
}

Key words: vibration system; energy decoupling; modal analysis; MATLAB; ADAMS

Abstract: In linear vibration system, it is difficult to determine the relationship between generalized coordinates and the natural frequency and to obtain the coupling relationship between generalized coordinates. Therefore, based on the MATLAB language the energy decoupling method is used to calculate the energy distribution matrix of the system, which obtains each order modal of the generalized coordinates and coupling relationship of generalized coordinates. Finally, car five degrees of freedom vibration system is established in the ADAMS to verify the calculation results.

\section{Introduction}

Newton-Euler method, Lagrange method and Kane's method ${ }^{[1]}$ are common to establish the dynamic equation that is a precondition of modal analysis. In fact, modal analysis is the most effective way to control vibration and noise ${ }^{[2]}$. For linear system, it is easy to obtain the frequencies and vibration modes, but it is difficult to determine that generalized coordinates belong to which the natural frequency, especially in the high degree of coupling between the system of generalized coordinates. There is no analysis of the natural frequency of the generalized coordinate in literature $^{[3-5]}$.

To this, this paper, taking a sort of car for example, does the following studies: the mechanics model of five degree of freedom is established; according to the Newton's method, the dynamic equation is established; the energy decoupling method ${ }^{[6-8]}$, the common methods in the powertrain mount system vibration isolation design, is used; each order modal energy distribution of generalized coordinates is obtained by using the MATLAB language, which illustrates the corresponding relationship between generalized coordinates and each order modal and coupling relationship between generalized coordinates; the modal analysis and calculation results based on energy decoupling method is verified by using the ADAMS.

\section{The modal analysis of car five degree of freedom vibration system}

\subsection{Mathematic model}

According to the feature of the car, the car is simplified a rigid body system that is made up the tires, suspension, bodywork and man-seat system, as shown in Fig.1. The system has five degrees of freedom. Generalized coordinates are $z_{i}(i=1,2,3,4,5)$ which respectively express vertical displacement of front-unsprung weight, vertical displacement of back-unsprung weight, vertical displacement of bodywork, angular displacement of bodywork and vertical displacement of man-seat system. $z_{g 1}$ and $z_{g 2}$ respectively express the roughness road excitation of the front and back wheels. A sort of car the kinetic parameters show in Table 1. 


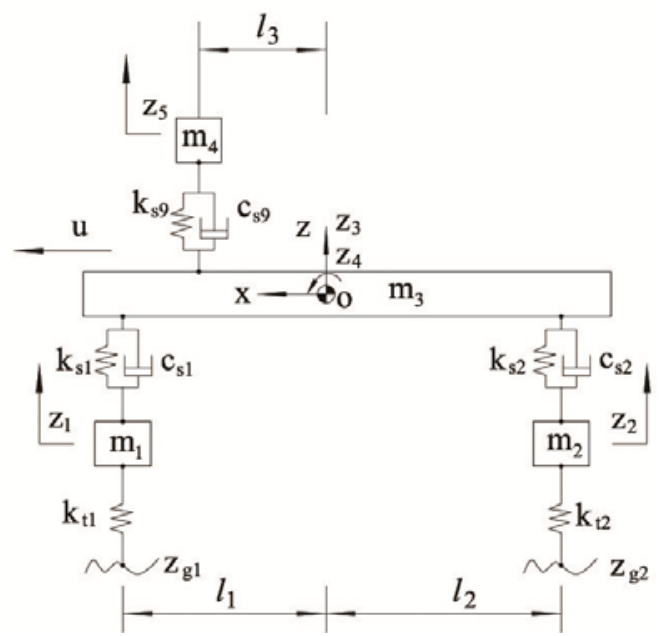

Fig. 1. The mechanical model of car five degree of freedom vibration system

\begin{tabular}{|c|c|c|c|}
\hline & Symbol (unit) & Numerical value & Explanation \\
\hline \multirow{5}{*}{$\begin{array}{l}\text { Physical } \\
\text { parameters }\end{array}$} & $m_{1}(\mathrm{~kg})$ & 40.5 & Front-unsprung mass \\
\hline & $m_{2}(\mathrm{~kg})$ & 45.4 & Back-unsprung mass \\
\hline & $m_{3}(\mathrm{~kg})$ & 700 & Bodywork mass \\
\hline & $J_{x x}\left(\mathrm{~kg} \cdot \mathrm{m}^{2}\right)$ & 2400 & $\begin{array}{l}\text { Moment of inertia of bodywork about the horizontal } \\
\text { axis }\end{array}$ \\
\hline & $m_{4}(\mathrm{~kg})$ & 80 & Man-seat mass \\
\hline \multirow{8}{*}{$\begin{array}{c}\text { Mechanical } \\
\text { parameter }\end{array}$} & $k_{t 1}(\mathrm{kN} / \mathrm{m})$ & 200 & Front wheel stiffness \\
\hline & $k_{t 2}(\mathrm{kN} / \mathrm{m})$ & 200 & Back wheel stiffness \\
\hline & $k_{\mathrm{s} 1}(\mathrm{kN} / \mathrm{m})$ & 24 & Front suspension stiffness \\
\hline & $k_{\mathrm{s} 2}(\mathrm{kN} / \mathrm{m})$ & 19 & Back suspension stiffness \\
\hline & $k_{\mathrm{s} 3}(\mathrm{kN} / \mathrm{m})$ & 5 & The seat stiffness \\
\hline & $C_{s 1}(\mathrm{kN} \cdot \mathrm{s} / \mathrm{m})$ & 1.6 & Front suspension damping \\
\hline & $c_{\mathrm{s} 2}(\mathrm{kN} \cdot \mathrm{s} / \mathrm{m})$ & 1.8 & Back suspension damping \\
\hline & $C_{\mathrm{s} 3}(\mathrm{kN} \cdot \mathrm{s} / \mathrm{m})$ & 0.72 & The seat damping \\
\hline \multirow{3}{*}{$\begin{array}{l}\text { Geometric } \\
\text { parameters }\end{array}$} & $l_{1}(\mathrm{~m})$ & 1.25 & The distance of body center of mass to the front axle \\
\hline & $l_{2}(\mathrm{~m})$ & 1.51 & The distance of body center of mass to the back axle \\
\hline & $l_{3}(\mathrm{~m})$ & 0.5 & $\begin{array}{c}\text { The distance of body center of mass to the center of } \\
\text { the seat }\end{array}$ \\
\hline
\end{tabular}

\subsection{The dynamic equation}

Because car five degree of freedom vibration system moves in the vertical plane and the system is relatively simple, using Newton's second law establishes the dynamic equation.

$$
\begin{gathered}
m_{1} \ddot{z}_{1}=-k_{t 1} z_{1}+k_{s 1}\left(z_{3}-z_{4} l_{1}-z_{1}\right)+c_{s 1}\left(\dot{z}_{3}-\dot{z}_{4} l_{1}-\dot{z}_{1}\right) \\
m_{2} \ddot{z}_{2}=-k_{t 2} z_{2}+k_{s 2}\left(z_{3}+z_{4} l_{1}-z_{2}\right)+c_{s 2}\left(\dot{z}_{3}+\dot{z}_{4} l_{2}-\dot{z}_{2}\right) \\
m_{3} \ddot{z}_{3}=-k_{s 1}\left(z_{3}-z_{4} l_{1}-z_{1}\right)-c_{s 1}\left(\dot{z}_{3}-\dot{z}_{4} l_{1}-\dot{z}_{1}\right)-k_{s 2}\left(z_{3}+z_{4} l_{2}-z_{2}\right)-c_{s 2}\left(\dot{z}_{3}+\right. \\
\left.\dot{z}_{4} l_{2}-\dot{z}_{2}\right)+k_{s 3}\left(z_{5}+z_{4} l_{3}-z_{3}\right)+c_{s 3}\left(\dot{z}_{5}+\dot{z}_{4} l_{3}-\dot{z}_{3}\right) \\
J_{x x} \ddot{z}_{4}=\left(k_{s 1}\left(z_{3}-z_{4} l_{1}-z_{1}\right)+c_{s 1}\left(\dot{z}_{3}-\dot{z}_{4} l_{1}-\dot{z}_{1}\right)\right) l_{1}-\left(k_{s 2}\left(z_{3}+z_{4} l_{2}-z_{2}\right)-c_{s 2}\left(\dot{z}_{3}+\right.\right. \\
\left.\left.\dot{z}_{4} l_{2}-\dot{z}_{2}\right)\right) l_{2}-\left(k_{s 3}\left(z_{5}+z_{4} l_{3}-z_{3}\right)+c_{s 3}\left(\dot{z}_{5}+\dot{z}_{4} l_{3}-\dot{z}_{3}\right)\right) l_{3} \\
m_{4} \ddot{z}_{5}=-k_{s 3}\left(z_{5}+z_{4} l_{3}-z_{3}\right)-c_{s 3}\left(\dot{z}_{5}+\dot{z}_{4} l_{3}-\dot{z}_{3}\right)
\end{gathered}
$$

Note: the reason why the original length of the spring and the weight of the parts itself are not considered when dynamics equation is established in this paper, because the generalized coordinates are relative to their static equilibrium position; this paper mainly studies the system free vibration and $z_{g 1}=z_{g 2}=0$. 
By combining and simplifying equation (1), (2), (3), (4) and (5), the equation (6) can be obtain:

$$
M \ddot{Z}+C \dot{Z}+K Z=0
$$

where,

$$
\begin{aligned}
& M=\left[\begin{array}{lllll}
m_{1} & & & & \\
& m_{2} & & & \\
& & m_{3} & & \\
& & & J X X & \\
& & & & m_{5}
\end{array}\right], \quad Z=\left(\begin{array}{lllll}
z_{1}, & z_{2}, & z_{3}, & z_{4}, & z_{5}
\end{array}\right)^{\mathrm{T}}
\end{aligned}
$$

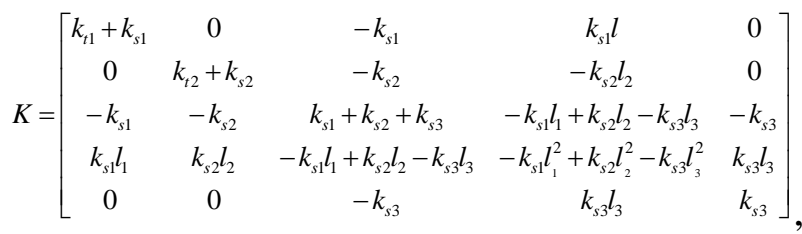

$$
\begin{aligned}
& \mathrm{C}=\left[\begin{array}{ccccc}
c_{\mathrm{s} 1} & 0 & -c_{s 1} & c_{s 1} l & 0 \\
0 & c_{\mathrm{s} 2} & -c_{s 2} & -c_{s 2} l_{2} & 0 \\
-c_{s 1} & -c_{s 1} & c_{s 1}+c_{s 2}+c_{s 3} & -c_{s 1} l_{1}+c_{s 2} l_{2}-c_{s 3} l_{3} & -c_{s 3} \\
c_{s 1} l_{1} & -c_{s 1} l_{2} & -c_{s 1} l_{1}+c_{s 2} l_{2}-c_{s 3} l_{3} & c_{s 1} l_{1}^{2}+c_{s 2} l_{2}^{2}+c_{s 3} l_{3}^{2} & c_{s 3} l_{3} \\
0 & 0 & -c_{s 3} & c_{s 3} l_{3} & c_{s 3}
\end{array}\right]
\end{aligned}
$$

\subsection{Natural frequency and modal matrix}

The focus of this paper is the undamped free vibration system, so the damping coefficient is 0 , and the equation (7) is obtained.

$$
M \ddot{Z}+C \dot{Z}=0
$$

According to vibration theory, by the generalized eigenvalue and generalized eigenvector of the matrix $\boldsymbol{K}$ and $\boldsymbol{M}$, diagonal matrix $\Lambda$ and modal matrix $\Phi$ are obtained. The $\Lambda$ which is the diagonal matrix corresponding to circular frequency squared is equivalent to diag $\left(\omega_{1}^{2}, \ldots, \omega_{5}^{2}\right.$,). The roots of the $\Lambda$ are circular frequencies of the vibration system, and the natural frequencies of the vibration system are obtained when the roots divides by $2 \pi$. Only a command in MATLAB, [V $\mathrm{D}]=\mathrm{eig}(\mathrm{K}, \mathrm{M})$, the modal matrix and diagonal matrix $\mathrm{D}$ can be get .

According to Table 1 and equation (7), the mass and stiffness matrix can be obtained. Using the MATLAB language programming, the modal matrix $\Phi$ and the diagonal matrix $\Lambda$ are obtained as follows:

$$
\Phi=\left[\begin{array}{ccccc}
-0.0030 & -0.0024 & 0.0032 & -0.0003 & 0.1571 \\
0.0024 & -0.0029 & 0.0020 & 0.1484 & 0.0003 \\
-0.0025 & -0.0269 & 0.0264 & -0.0008 & -0.0010 \\
0.0199 & -0.0040 & -0.0022 & -0.0004 & 0.0004 \\
-0.0239 & -0.0753 & -0.0791 & 0.0000 & 0.0000
\end{array}\right], \Lambda=\left[\begin{array}{ccccc}
29.8 & & & & \\
& 41.8 & & & \\
& & 84.2 & & \\
& & & 4827.7 & \\
& & & & 5536.3
\end{array}\right]
$$

Based on the diagonal matrix, the natural frequencies can be obtained:

$f_{1}=0.8695 \mathrm{~Hz}, \quad f_{2}=1.0295 \mathrm{~Hz}, \quad f_{3}=1.4605 \mathrm{~Hz}, \quad f_{4}=11.0584 \mathrm{~Hz}, \quad f_{5}=11.8421 \mathrm{~Hz}$

The eig function of MATLAB language is used, and the natural frequency will be arranged from small to large order. So it is hard to learn the generalized coordinates of the each natural frequency. Using the method, by simply observing the biggest elements in each column of modal matrix, obtains the greatest contribution of the generalized coordinates. Because the method does not consider the mass, unit and plus or minus, it is not reasonable. The method could have an error in judgment when high coupling of the generalized coordinates. To this, the problem will be solved by energy method in the next. 


\section{The energy distribution of car five degree of freedom vibration system}

\subsection{Energy decoupling}

Vibration system usually exists in different degrees of coupling. The effect can make the system vibration band widened, which brings difficulty to vibration isolation and frequency allocation. The percentage of a generalized coordinates in one order modal total kinetic energy is selected as evaluation index of modal decoupling, which names energy method of decoupling.

According to the equation (7), circular frequency and modal matrix can be obtained, and the system energy distribution of each order main vibration can be calculated. The energy distribution written in matrix form is called the energy distribution matrix.

The maximum kinetic energy of the i-th order principal vibration can be expressed as follows:

$$
T_{\max }^{i}=\frac{1}{2} \omega_{i}^{2} \phi_{i}^{t} M \phi_{i}
$$

The expansion of the equation (8) is expressed as follows:

$$
T_{\max }^{i}=\frac{1}{2} \omega_{i}^{2} \sum_{l=1}^{5} \sum_{k=1}^{5}\left(\phi_{i}\right)_{l}\left(\phi_{i}\right)_{k} m_{k l}
$$

In the above equation, $m_{k l}$ expresses the k-th row and l-th column of mass matrix, and $\left(\phi_{i}\right)_{k}$ and $\left(\phi_{i}\right)_{l}$ respectively express the k-th element and the l-th element of the modal $\left(\phi_{i}\right)$.

The percentage of the $\mathrm{k}$-th generalized coordinates in the i-th order modal total kinetic energy is the following euqation:

$$
T_{p}(k, i)=\frac{T_{k}}{T_{\max }^{i}}=\frac{\sum_{l=1}^{5}\left(\phi_{i}\right)_{l}\left(\phi_{i}\right)_{k} m_{k l}}{\sum_{l=1}^{5} \sum_{k=1}^{5}\left(\phi_{i}\right)_{l}\left(\phi_{i}\right)_{k} m_{k l}} \times 100 \%
$$

If $T_{p}(k, i)$ is equivalent to $100 \%$, it means that the energy of the i-th order principal modes completely focuses on the k-th generalized coordinates, and the energy of the rest of the generalized coordinates assigned is zero. The case illustrates that there is no coupling of the principal vibration corresponding to the order modal.

Based on the theory of energy decoupling method, the function matrix_of_enegy is developed by using the MATLAB language. The input parameters the function are modal matrix V, diagonal matrix $\mathrm{D}$ and mass matrix $\mathrm{M}$, and the output parameter is energy distribution matrix Tp. The call format is as follows:

$$
\text { Tp=matrix_of_enegy(V,D,M) }
$$

3.2 Energy distribution matrix of car five degree of freedom vibration system Base on section 2.1, by using the function of energy decoupling method, modal matrix, diagonal matrix and mass matrix, it is easy to obtain the energy distribution of the system as shown in Table 2.

Table 2. The natural frequency and energy distribution of the vibration system

\begin{tabular}{cccccc}
\hline \multirow{2}{*}{\begin{tabular}{c} 
The \\
energy \\
distribution \\
\cline { 2 - 6 } $\begin{array}{c}\text { in the five } \\
\text { directions }\end{array}$
\end{tabular}} & 0.8695 & 1.0295 & 1.4605 & 11.0584 & 11.8421 \\
\hline$Z_{1}$ & 0.0353 & 0.0226 & 0.0406 & 0.0004 & $\mathbf{9 9 . 9 0 1 1}$ \\
$Z_{2}$ & 0.0261 & 0.0378 & 0.0189 & $\mathbf{9 9 . 9 1 6 8}$ & 0.0003 \\
$Z_{3}$ & 0.4544 & $\mathbf{5 0 . 6 7 5 1}$ & 48.7526 & 0.0498 & 0.0681 \\
$Z_{4}$ & $\mathbf{9 4 . 9 1 0 5}$ & 3.8849 & 1.1411 & 0.0329 & 0.0305 \\
$Z_{5}$ & 4.5737 & 45.3796 & $\mathbf{5 0 . 0 4 6 8}$ & 0.0000 & 0.0000 \\
\hline
\end{tabular}


From Table 2 we can find that the energies of $Z_{4}, Z_{3}, Z_{5}, Z_{2}$ and $Z_{1}$ respectively reach maximum from first to fifth order modal, which illustrates the coupling relationship between different generalized coordinates corresponding to natural frequencies. Also we can find that the coupling of $Z_{3}$ and $Z_{5}$ are high in the second and third order modal.

\section{The modal verification of car five degree of freedom vibration system}

\subsection{The ADAMS model of car five degree of freedom vibration system}

Multi-body system dynamics software ADAMS is authority in mechanical dynamics analysis. Then this paper by using ADAMS verifies the simulation results by MATLAB. The ADAMS model of car five degree of freedom vibration system is established, as shown in Fig. 2.

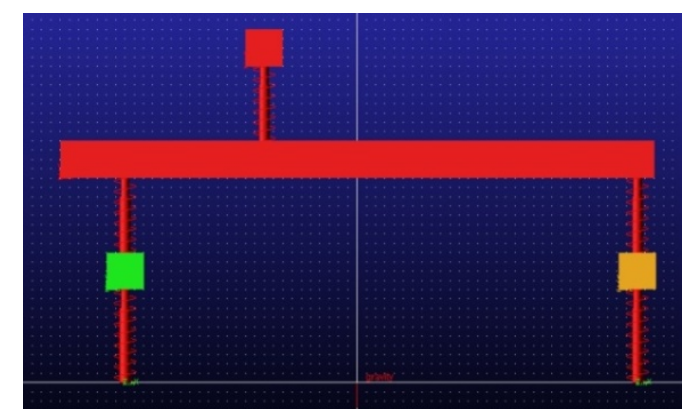

Fig.2. The ADAMS model of car five degree of freedom vibration system

\subsection{The natural frequency comparison}

The natural frequency comparison of the system solved by MATLAB and ADAMS is as shown in Table 3. The table expresses the calculation results of the natural frequency as to car five degree of freedom undamped vibration system. From Table 3 we can find that the calculation results of the natural frequency solved by MATLAB is the same as ADAMS. Therefore, it illustrates that the modeling method and results of this paper are accurate and reliable.

Table 3. The natural frequency comparison of the system solved by

\begin{tabular}{cccccc}
\multicolumn{5}{c}{ MATLAB and ADAMS } \\
\hline MATLAB & 0.8695 & 1.0295 & 1.4605 & 11.0584 & 11.8421 \\
ADAMS & 0.8695 & 1.0295 & 1.4605 & 11.0584 & 11.8421 \\
\hline
\end{tabular}

\subsection{ADAMS mode shape}

The modal shapes of car five degree of freedom vibration system in ADAMS is as shown in Fig.3. From Fig. 3 we can find that the first modal mainly focuses on $Z_{4}$, the second and third modals mainly focus on $Z_{3}$ and $Z_{5}$, the fourth modal mainly focuses on $Z_{2}$, and the fifth modal mainly focuses on $Z_{4}$. Fig.3 is extremely consistent with the energy distribution in Table 2. Once again Fig. 3 illustrates that simulation results of this paper are accurate and reliable.

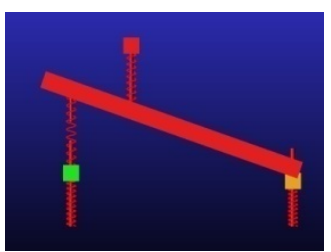

(a)

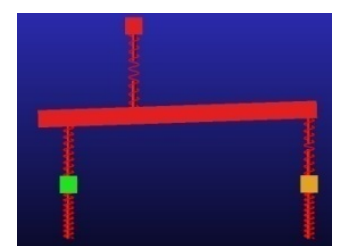

(b)

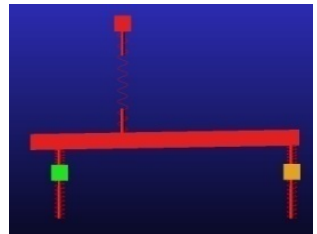

(c)

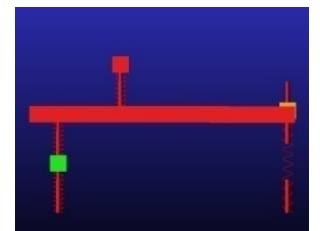

(d)

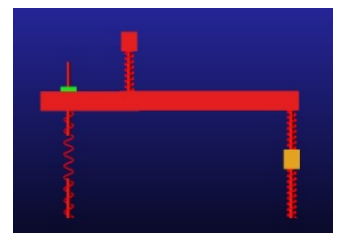

(e)

Fig.3. The modal shapes of car five degree of freedom vibration system in ADAMS 
Note: the figure (a), (b), (c), (d) and (e) respectively express the modal shapes corresponding to the natural frequencies $\mathrm{f} 1, \mathrm{f} 2, \mathrm{f} 3, \mathrm{f} 4$ and $\mathrm{f} 5$.

\section{Conclusion}

Taking the car five degree of freedom vibration system as the object of study, this paper makes modal analysis. The energy distribution of the vibration system, which is used to illustrate the corresponding relationship between generalized coordinates and each order modal and coupling relationship between generalized coordinate, is obtained base on the energy decoupling method. Last, using ADAMS verifies the simulation results solved by MATLAB, which lays the foundation of car vibration isolation and optimization.

\section{Acknowledgements}

This paper was supported by Guangxi University of science and technology research projects (NO.KY2015YB101 and NO.KY2015YB102), the Guangxi Key Laboratory of Manufacturing Systems and Advanced Manufacturing Technology (No.13-051-09-009Z and No.14-045-15-008Z).

\section{Reference}

[1] LIU Yanzhu. Advanced dynamics [M]. Bejing: HIGHER EDUCATION PRESS, 2001.

[2] LI Wei, SHI Wenku. Application of modal analysis in the modification of light vehicle[J]. Noise and Vibration Control. 2008, 28(04): 75-78.

[3] ZHU Weiyu. Ride performance of heavy vehicle and the suspension damper optimal design [D]. Hunan University, 2012.

[4] LI Beibei. Modeling, simulation and experiment study on ride performance of light-duty trucks[D]. Shandong University, 2008.

[5] QIAN Kai. Vehicle ride comfort analysis and particle swarm optimization in the application of suspension[D]. Chongqing Jiaotong University, 2013.

[6] YAN Hongyu, XU Shian. Energy method decoupling and computer optmization engine mounting systems[J]. Automotive Engineering, 1993, 15(06): 321-328.

[7] SUN Beibei, ZHANG Qijun, SUN Qinghong, etal. Study on decoupled engine mounting system[J]. JOURNAL OF VIBRATION ENGINEERING. 1994, 7(03): 240-245.

[8] LONG Yan, SHI Wenku, JIANG Lingshan, etal. A comparative study on the optimization methods for powertrain mounting system[J]. Automotive Engineering. 2011, 33(10): 875-879. 